\title{
INTRODUCTION OF A BLOCK TROLLEY = A MOBILE BLOCK ROOM
}

\author{
A. Sadeghi, A. Tay, S. Haddad, S. Natarajan, B. Bellew \\ St Mary's Hospital, Anaesthetics, London, United Kingdom
}

\section{Background and Aims:}

Regional anaesthesia has demonstrated numerous benefits including better analgesic outcomes than general anaesthesia with systemic opioids, decreased post-operative nausea and vomiting rates \& increased patient satisfaction. As a result we have seen a surge in popularity; in particular with the widespread availability of ultrasound imaging. ${ }^{1}$

Despite this, obstacles cited include anaesthetic room delays which often hinder the routine use of such techniques. Thus creating an area with access to all the appropriate equipment would help to overcome this. ${ }^{2}$

\section{Methods:}

The current economic climate and space limitations often prevent the creation of designated "block rooms" and thus we created a "block trolley". Containing all necessary drugs and equipment in one location, this essentially creates a mobile block room that can be taken to any clinical area.

Surveys were taken of consultant anaesthetists and operating room practitioners (ODPs) at our trauma centre before and after trolley implementation to assess the impact on provision of regional anaesthesia.
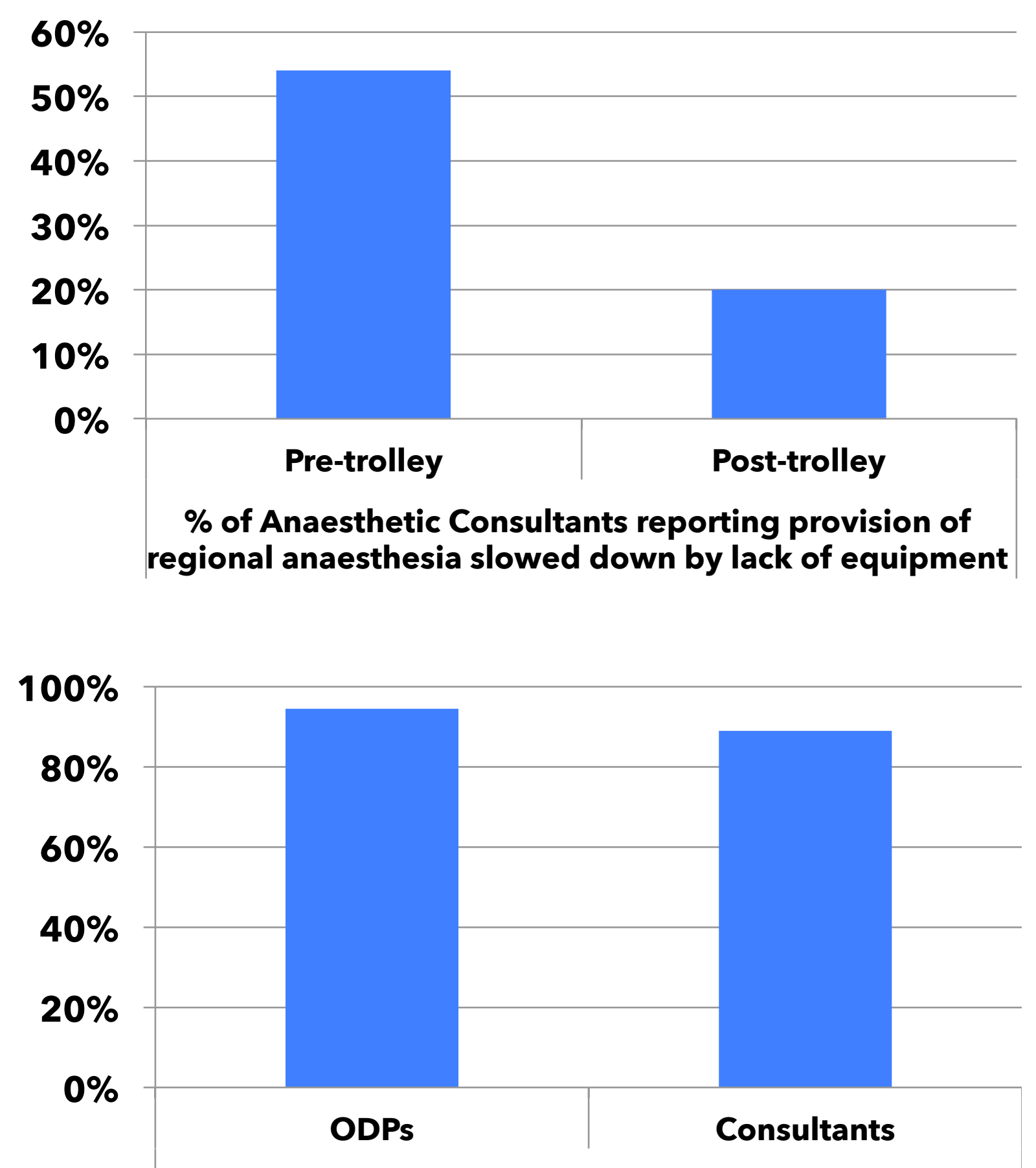

Was the introduction of the block trolley useful?

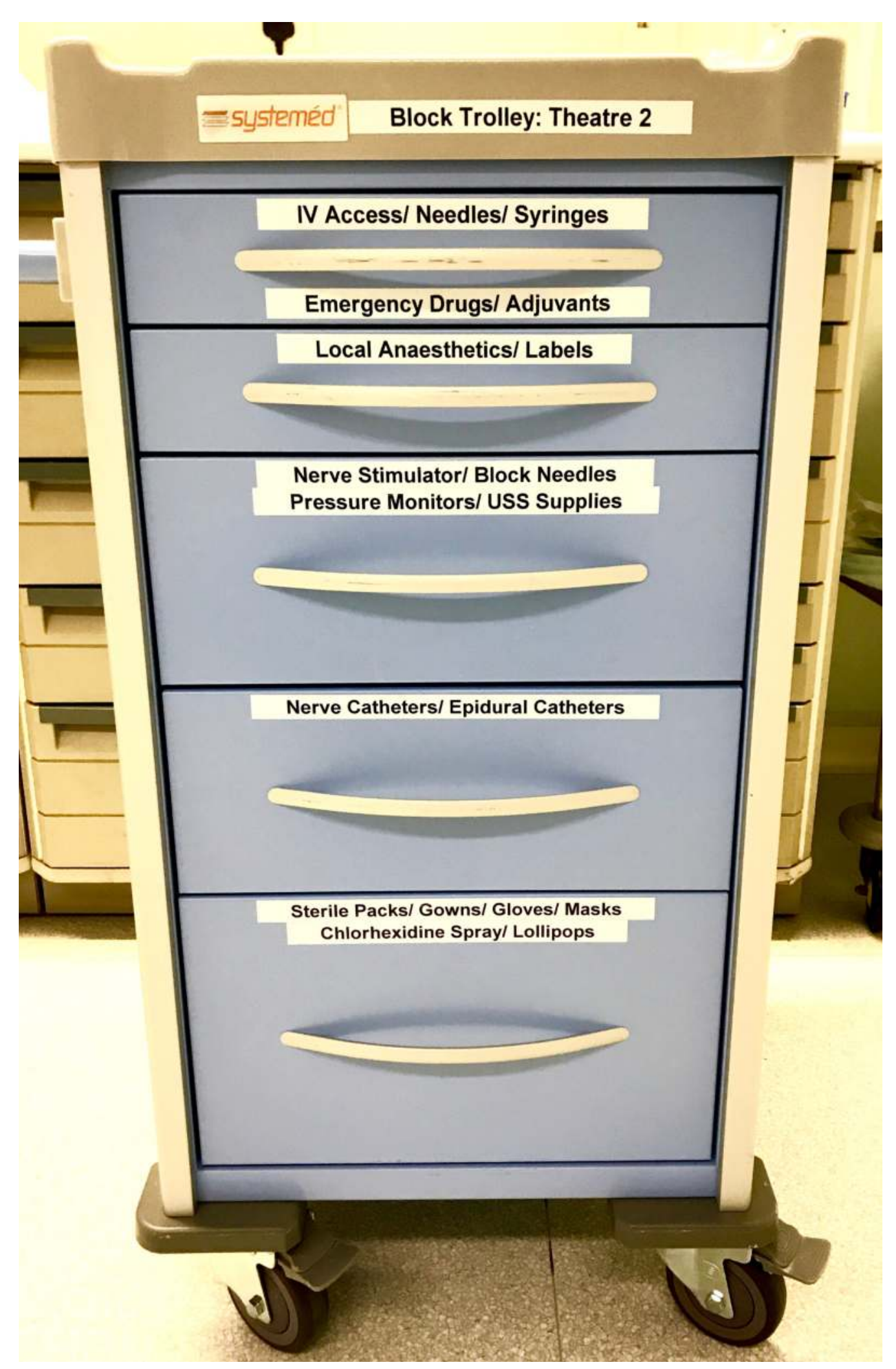

\section{Results:}

Pre-introduction surveys indicated that that $54 \%$ of ODPs and consultants stated "lack of equipment" slowed down the provision of regional anaesthesia and $86 \%$ of ODPs and $67 \%$ of consultants felt the introduction of a "block trolley" would be useful.

The introduction of the trolley put all equipment/drugs in an accessible location and consequently only $20 \%$ of consultants stated that they were slowed down by lack of equipment on postintroduction surveys. In addition, $89 \%$ of consultants and $95 \%$ of ODPs who had the trolley stated it had been useful

\section{Conclusions:}

Implementation of "Block trolleys" may improve accessibility to regional anaesthesia in departments without the space or resources for a designated block room.

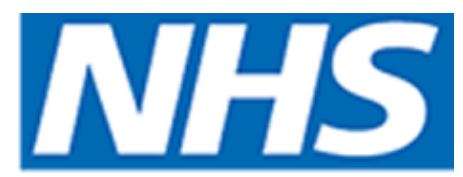

\section{Imperial College Healthcare} NHS Trust 